\title{
The Romanoff theorem revisited
}

by

\section{Hongze Li (Shanghai) and HaO PAN (Nanjing)}

For a subset $A$ of positive integers, define $A(x)=|\{1 \leq a \leq x: a \in A\}|$. Let $\mathcal{P}$ denote the set of all primes and $2^{\mathbb{N}}=\left\{2^{n}: n \in \mathbb{N}\right\}$, where $\mathbb{N}=$ $\{0,1,2, \ldots\}$. A classical result of Romanoff [6] asserts that the sumset

$$
2^{\mathbb{N}}+\mathcal{P}=\left\{2^{n}+p: n \in \mathbb{N}, p \in \mathcal{P}\right\}
$$

has a positive lower density, i.e., there exists a positive constant $C_{R}$ such that $\left(2^{\mathbb{N}}+\mathcal{P}\right)(x) \geq C_{R} x$ for sufficiently large $x$. Recently, the lower bound of $C_{R}$ has been calculated in $[2,3,5]$. Now let

$$
\mathcal{P}_{2}=\{q: q \text { is a prime or the product of two primes }\} .
$$

Motivated by Romanoff's theorem, in this short note we shall show that:

THEOREM 1. The sumset

$$
2^{\mathcal{P}}+\mathcal{P}_{2}=\left\{2^{p}+q: p \in \mathcal{P}, q \in \mathcal{P}_{2}\right\}
$$

has a positive lower density.

Proof. In our proof, the constants implied by $\ll, \gg$ and $O(\cdot)$ will be always absolute.

For $q \in \mathcal{P}_{2} \backslash \mathcal{P}$, let $\psi(q)$ be the least prime factor of $q$. Let

$$
\mathcal{P}_{2}^{*}=\left\{q \in \mathcal{P}_{2} \backslash \mathcal{P}: \psi(q)<q^{1 / 3}\right\} .
$$

It suffices to show that $2^{\mathcal{P}}+\mathcal{P}_{2}^{*}$ has a positive lower density.

By the Chebyshev theorem, we have

$$
\frac{x}{5 \log x} \leq \mathcal{P}(x) \leq \frac{5 x}{\log x} .
$$

2000 Mathematics Subject Classification: Primary 11P32; Secondary 11B05, 11N36.

Key words and phrases: positive lower density, primes, powers of 2 .

This work was supported by the National Natural Science Foundation of China (Grant No. 10771135). The second author is the corresponding author. 
Hence for $x \geq e^{750}$,

$$
\begin{aligned}
\mathcal{P}_{2}^{*}(x) & =\left|\left\{\left(p_{1}, p_{2}\right): p_{1}, p_{2} \in \mathcal{P}, p_{1}^{2}<p_{2} \leq x / p_{1}\right\}\right| \\
& \geq \sum_{\substack{p_{1} \in \mathcal{P} \\
p_{1} \leq x^{1 / 3}}}\left(\frac{x / p_{1}}{5 \log \left(x / p_{1}\right)}-\frac{5 p_{1}^{2}}{\log \left(p_{1}^{2}\right)}\right) \\
& \geq \frac{x}{5 \log x} \sum_{\substack{p_{1} \in \mathcal{P} \\
p_{1} \leq x^{1 / 3}}} \frac{1}{p_{1}}-\frac{5 x^{1 / 3}}{\log \left(x^{1 / 3}\right)} \cdot \frac{5 x^{2 / 3}}{\log \left(x^{2 / 3}\right)} \\
& \geq \frac{x \log \log x}{10 \log x},
\end{aligned}
$$

since (cf. [1, Theorem 8.8.5])

$$
\log \log x \leq \sum_{p \in \mathcal{P} \cap[1, x]} \frac{1}{p} \leq \log \log x+C,
$$

where $C$ is an absolute constant.

Similarly it is not difficult to deduce that $\mathcal{P}_{2}^{*}(x) \ll x \log \log x / \log x$. Let

$$
r(n)=\left|\left\{(p, q): n=2^{p}+q, p \in \mathcal{P}, q \in \mathcal{P}_{2}^{*}\right\}\right| .
$$

Clearly we have

$$
\begin{aligned}
\sum_{n \leq x} r(n) & =\left|\left\{(p, q): p \in \mathcal{P}, q \in \mathcal{P}_{2}^{*}, 2^{p}+q \leq x\right\}\right| \\
& \geq 2^{\mathcal{P}}(x / 2) \mathcal{P}_{2}^{*}(x / 2) \\
& \gg \frac{\log x}{\log \log x} \cdot \frac{x \log \log x}{\log x}=x .
\end{aligned}
$$

And by Cauchy-Schwarz's inequality,

$$
\left(\sum_{n \leq x} r(n)\right)^{2} \leq\left(2^{\mathcal{P}}+\mathcal{P}_{2}^{*}\right)(x) \sum_{n \leq x} r(n)^{2} .
$$

Therefore we only need to prove that

$$
\begin{aligned}
& \sum_{n \leq x} r(n)^{2} \\
& \quad=\left|\left\{\left(p_{1}, p_{2}, q_{1}, q_{2}\right): p_{1}, p_{2} \in \mathcal{P}, q_{1}, q_{2} \in \mathcal{P}_{2}^{*}, 2^{p_{1}}+q_{1}=2^{p_{2}}+q_{2} \leq x\right\}\right|
\end{aligned}
$$
is $O(x)$.

Below we shall show that

$$
\left|\left\{q \leq x-N: q, q+N \in \mathcal{P}_{2}^{*}\right\}\right| \ll \frac{x(\log \log x)^{2}}{(\log x)^{2}} \prod_{p \mid N}\left(1+\frac{1}{p}\right)
$$


for each positive even integer $N$. Define

$$
\mathfrak{S}(n)=\prod_{p \mid n}\left(1+\frac{1}{p}\right) .
$$

Suppose that $k_{1}, k_{2}, l_{1}, l_{2}$ are positive integers such that $\left(k_{i}, l_{i}\right)=1$ and $2 \mid k_{2} l_{1}-k_{1} l_{2}$. Let

$$
\mathscr{A}=\left\{\left(k_{1} n+l_{1}\right)\left(k_{2} n+l_{2}\right): 1 \leq n \leq x\right\}
$$

and $\mathscr{A}_{d}=\{a \in \mathscr{A}: d \mid a\}$. Then for any square-free $d$,

$$
\mathscr{A}_{d}=\frac{\omega(d)}{d} x+O(\omega(d)),
$$

where $\omega(d)$ is a multiplicative function such that for a prime $p$,

$$
\omega(p)= \begin{cases}2 & \text { if } p \nmid k_{1} k_{2}\left(k_{2} l_{1}-k_{1} l_{2}\right), \\ 1 & \text { if } p \nmid k_{1} k_{2} \text { and } p \mid\left(k_{2} l_{1}-k_{1} l_{2}\right), \\ & \text { or } p \mid k_{1} \text { and } p \nmid k_{2}, \text { or } p \mid k_{2} \text { and } p \nmid k_{1}, \\ 0 \quad & \text { if } p \mid k_{1} \text { and } p \mid k_{2} .\end{cases}
$$

As an application of Selberg's sieve method (cf. [4, Sections 7.2 and 7.3]), we know that

$$
\left|\left\{1 \leq n \leq x: k_{1} n+l_{1}, k_{2} n+l_{2} \in \mathcal{P}\right\}\right| \ll \frac{x}{(\log x)^{2}} \mathfrak{S}\left(k_{1} k_{2}\right) \mathfrak{S}\left(k_{2} l_{1}-k_{1} l_{2}\right) .
$$

Observe that $n, n+N \in \mathcal{P}_{2} \backslash \mathcal{P}$ if and only if there exist $p_{1}, p_{2} \in \mathcal{P}$ such that $n / p_{1},(n+N) / p_{2} \in \mathcal{P}$. Assume that $n / p_{1}=p_{2} m+l$ where $1 \leq l \leq p_{2}$. Then

$$
(n+N) / p_{2}=\left(p_{1} p_{2} m+p_{1} l+N\right) / p_{2}=p_{1} m+\left(p_{1} l+N\right) / p_{2},
$$

whence $p_{1} l \equiv-N\left(\bmod p_{2}\right)$. Note that $l$ is uniquely determined by $p_{1}$ and $p_{2}$ unless $p_{1}=p_{2}$. Thus

$$
\begin{aligned}
\mid\{n \leq x & \left.: n, n+N \in \mathcal{P}_{2}^{*}, p_{1}\left|n, p_{2}\right|(n+N)\right\} \mid \\
& \leq \begin{cases}\left|\left\{m \leq x / p_{1}: m, m+N / p_{1} \in \mathcal{P}\right\}\right| & \text { if } p_{1}=p_{2}, \\
\left|\left\{m \leq x / p_{1} p_{2}: p_{2} m+l, p_{1} m+\left(p_{1} l+N\right) / p_{2} \in \mathcal{P}\right\}\right| & \text { otherwise, }\end{cases} \\
& \ll \begin{cases}\frac{x / p_{1}}{\left(\log \left(x / p_{1}\right)\right)^{2}} \mathfrak{S}\left(N / p_{1}\right) & \text { if } p_{1}=p_{2} \mid N, \\
\frac{x / p_{1} p_{2}}{\left(\log \left(x / p_{1} p_{2}\right)\right)^{2}} \mathfrak{S}\left(p_{1} p_{2}\right) \mathfrak{S}(N) & \text { otherwise. }\end{cases}
\end{aligned}
$$

Therefore

$$
\begin{aligned}
& \left|\left\{q \leq x-N: q, q+N \in \mathcal{P}_{2}^{*}\right\}\right| \\
& \quad \ll \sum_{\substack{p_{1}, p_{2} \in \mathcal{P} \\
p_{1}, p_{2} \leq x^{1 / 3}}} \frac{x / p_{1} p_{2}}{\left(\log \left(x / p_{1} p_{2}\right)\right)^{2}} \mathfrak{S}\left(p_{1} p_{2}\right) \mathfrak{S}(N)+\sum_{\substack{p \in \mathcal{P} \\
p \mid N, p \leq x^{1 / 3}}} \frac{x / p}{(\log (x / p))^{2}} \mathfrak{S}(N / p) .
\end{aligned}
$$


Now

$$
\begin{aligned}
\sum_{\substack{p_{1}, p_{2} \in \mathcal{P} \\
p_{1}, p_{2} \leq x^{1 / 3}}} \frac{x / p_{1} p_{2}}{\left(\log \left(x / p_{1} p_{2}\right)\right)^{2}}\left(1+\frac{1}{p_{1}}\right)\left(1+\frac{1}{p_{2}}\right) \\
\leq \frac{36 x}{(\log x)^{2}} \sum_{\substack{p_{1}, p_{2} \in \mathcal{P} \\
p_{1}, p_{2} \leq x^{1 / 3}}} \frac{1}{p_{1} p_{2}} \ll \frac{x(\log \log x)^{2}}{(\log x)^{2}} .
\end{aligned}
$$

And

$$
\sum_{\substack{p \in \mathcal{P} \\ p \mid N, p \leq x^{1 / 3}}} \frac{x / p}{(\log (x / p))^{2}} \leq \sum_{\substack{p \in \mathcal{P} \\ p \leq x^{1 / 3}}} \frac{x / p}{(\log (x / p))^{2}} \ll \frac{x \log \log x}{(\log x)^{2}} .
$$

This concludes the proof of (2).

Let us return to the proof of (1). Clearly

$$
\sum_{n \leq x} r(n)^{2} \leq 2 \sum_{\substack{p_{1}, p_{2} \in \mathcal{P} \\ p_{2} \leq p_{1} \leq \log x / \log 2}}\left|\left\{q_{1} \in \mathcal{P}_{2}^{*}: 2^{p_{1}}-2^{p_{2}}+q_{1} \in \mathcal{P}_{2}^{*} \cap[1, x]\right\}\right| .
$$

If $p_{1}=p_{2}$, then

$$
\sum_{q_{1} \in \mathcal{P}_{2}^{*} \cap[1, x]}\left|\left\{q_{2} \in \mathcal{P}_{2}^{*} \cap[1, x]: q_{2}=2^{p_{1}}-2^{p_{2}}+q_{1}\right\}\right|=\mathcal{P}_{2}^{*}(x) \ll \frac{x \log \log x}{\log x} .
$$

And if $p_{1}>p_{2}$, then

$$
\begin{aligned}
\sum_{q_{1} \in \mathcal{P}_{2}^{*} \cap[1, x]} \mid\left\{q_{2} \in \mathcal{P}_{2}^{*} \cap[1, x]: q_{2}\right. & \left.=2^{p_{1}}-2^{p_{2}}+q_{1}\right\} \mid \\
& \ll \frac{x(\log \log x)^{2}}{(\log x)^{2}} \prod_{p \mid\left(2^{\left.p_{1}-p_{2}-1\right)}\right.}\left(1+\frac{1}{p}\right) .
\end{aligned}
$$

Hence we have

$$
\begin{aligned}
& \sum_{n \leq x} r(n)^{2} \ll \mathcal{P}\left(\frac{\log x}{\log 2}\right) \frac{x \log \log x}{\log x}+\frac{x(\log \log x)^{2}}{(\log x)^{2}} \sum_{\substack{p_{1}, p_{2} \in \mathcal{P} \\
p_{2}<p_{1} \leq \frac{\log x}{\log 2}}} \prod_{\left(2^{\left.p_{1}-p_{2}-1\right)}\right.}\left(1+\frac{1}{p}\right) \\
& \ll \frac{\log x}{\log \log x} \cdot \frac{x \log \log x}{\log x}+\frac{x(\log \log x)^{2}}{(\log x)^{2}} \sum_{0<k \leq \frac{\log x}{\log 2}} 2 \prod_{p \mid\left(2^{k}-1\right)}\left(1+\frac{1}{p}\right) \sum_{\substack{p_{1}, p_{2} \in \mathcal{P} \\
p_{2}<p_{1} \leq \frac{\log x}{\log 2}}} 1 \\
& p_{1}-p_{2}=k \\
& \ll x+\frac{x(\log \log x)^{2}}{(\log x)^{2}} \cdot \frac{2 \log x}{(\log \log x)^{2}} \sum_{0<k \leq \frac{\log x}{\log 2}} \prod_{p \mid\left(2^{k}-1\right)}\left(1+\frac{1}{p}\right) \prod_{p \mid k}\left(1+\frac{1}{p}\right) .
\end{aligned}
$$


For any positive odd integer $d$, let $e(d)$ denote the least positive integer such that $2^{e(d)} \equiv 1(\bmod d)$. Then $2^{k} \equiv 1(\bmod d)$ if and only if $e(d) \mid k$. Now

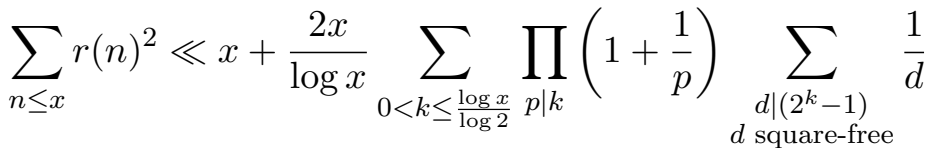

$$
\begin{aligned}
& =x+\frac{2 x}{\log x} \sum_{\substack{\text { square-free } \\
2 \nmid d}} \frac{1}{d} \sum_{\substack{0<k \leq \frac{\log x}{\log 2} \\
e(d) \mid k}} \prod_{p \mid k}\left(1+\frac{1}{p}\right) \\
& =x+\frac{2 x}{\log x} \sum_{\substack{d \text { square-free } \\
2 \nmid d}} \frac{1}{d} \sum_{\substack{d^{\prime} \\
\text { square-free }}} \frac{1}{d^{\prime}} \sum_{\substack{0<k \leq \log x \\
\log 2 \\
e(d)\left|k, d^{\prime}\right| k}} 1 \\
& \leq x+\frac{2 x}{\log x} \cdot \frac{\log x}{\log 2} \sum_{d, d^{\prime}} \frac{1}{\substack{\text { square-free } \\
2 \nmid d}} \frac{1}{d d^{\prime}\left[e(d), d^{\prime}\right]} \text {. }
\end{aligned}
$$

Our final task is to show that the series

$$
\sum_{\substack{d, d^{\prime} \text { square-free } \\ 2 \nmid d}} \frac{1}{d d^{\prime}\left[e(d), d^{\prime}\right]}
$$

converges. Clearly

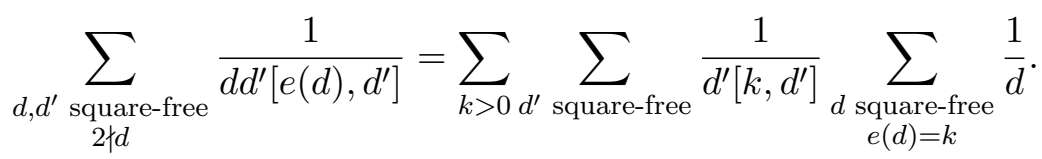

Let

$$
W(x)=\sum_{0<k \leq x} \sum_{\substack{\text { square-free } \\ e(d)=k}} \frac{1}{d} .
$$

With the help of the arguments of Romanoff (cf. [6], [4, p. 201]), we know that $W(x) \ll \log x$. And

$$
\begin{aligned}
\sum_{d^{\prime} \text { square-free }} \frac{1}{d^{\prime}\left[k, d^{\prime}\right]} & =\frac{1}{k} \prod_{p \in \mathcal{P}, p \mid k}\left(1+\frac{1}{p}\right) \prod_{p \in \mathcal{P}, p \nmid k}\left(1+\frac{1}{p^{2}}\right) \\
& \ll \frac{1}{k} \prod_{p \in \mathcal{P}, p \mid k}\left(1+\frac{1}{p}\right) \leq \frac{1}{\phi(k)} \ll k^{-2 / 3} .
\end{aligned}
$$


Hence

$$
\begin{aligned}
\sum_{\substack{d, d^{\prime} \\
\text { square-free } \\
2 \nmid d}} \frac{1}{d d^{\prime}\left[e(d), d^{\prime}\right]} & \ll \int_{1 / 2}^{\infty} x^{-2 / 3} d W(x)=\int_{1 / 2}^{\infty} \frac{2 W(x)}{3 x^{5 / 3}} d x+O(1) \\
& \ll \int_{1 / 2}^{\infty} \frac{\log x}{x^{5 / 3}} d x+O(1) \ll 1 .
\end{aligned}
$$

This completes the proof.

Remark. Professor Y.-G. Chen communicated to the second author the following two conjectures:

Conjecture 1. Let $A$ and $B$ be two sets of positive integers. If there exists a constant $c>0$ such that $A(\log x / \log 2) B(x)>c x$ for all sufficiently large $x$, then the set $\left\{2^{a}+b: a \in A, b \in B\right\}$ has a positive lower asymptotic density.

Conjecture 2. Let $A$ and $B$ be two sets of positive integers. If there exists a constant $c>0$ such that $A(\log x / \log 2) B(x)>c x$ for infinitely many positive integers $x$, then the set $\left\{2^{a}+b: a \in A, b \in B\right\}$ has a positive upper asymptotic density.

Acknowledgements. We are grateful to the anonymous referee for his/her useful suggestions on this paper. We also thank Professor Yong-Gao Chen for helpful discussions.

\section{References}

[1] E. Bach and J. Shallit, Algorithmic Number Theory, Foundations of Computing. Volume I: Efficient Algorithms, The MIT Press, Cambridge, MA, 1996.

[2] Y.-G. Chen and X.-G. Sun, On Romanoff's constant, J. Number Theory 106 (2004), $275-284$.

[3] L. Habsieger and X.-F. Roblot, On integers of the form $p+2^{k}$, Acta Arith. 122 (2006), 45-50.

[4] M. B. Nathanson, Additive Number Theory: Inverse Problems and the Geometry of Sumsets, Grad. Texts in Math. 165, Springer, New York, 1996.

[5] J. Pintz, A note on Romanov's constant, Acta Math. Hungar. 112 (2006), 1-14.

[6] N. P. Romanoff, Über einige Sätze der additiven Zahlentheorie, Math. Ann. 109 (1934), 668-678.

Department of Mathematics

Shanghai Jiaotong University

Shanghai 200240

People's Republic of China

E-mail: lihz@sjtu.edu.cn
Department of Mathematics

Nanjing University

Nanjing 210093

People's Republic of China

E-mail: haopan79@yahoo.com.cn

Received on 21.9.2007

and in revised form on 12.8.2008 